\title{
THE TOTAL ABSOLUTE CURVATURE OF CLOSED CURVES IN RIEMANNIAN MANIFOLDS
}

\author{
F. BRICKELL \& C. C. HSIUNG
}

\section{Introduction}

This paper is concerned with some extensions of the theorems of Fenchel [3], Milnor [5], Fary [2] on the total absolute curvature of closed curves in euclidean space. We obtain a theorem for closed curves in a complete simply connected riemannian $n$-manifold with nonpositive sectional curvature, and this leads to more precise results when the curvature is constant. Throughout this paper the summation convention for repeated indices is used, and all indices take the values $1, \cdots, n$ unless stated otherwise.

\section{Some geometry of shells}

Let 0 be a point on a closed $C^{\infty}$ curve $C$ embedded in a riemannian $n$ manifold $M$, and suppose that $C$ lies in a normal neighborhood of 0 . Then $C$ can be expressed in terms of its arc length $s$ as

$$
\exp _{0} r(s) \zeta(s), \quad 0<s<L
$$

where $r(s)>0, \zeta(s)$ is a unit vector in the tangent space $T_{0} M$, and $L$ is the total length of $C$. The functions $r, \zeta$ are $C^{\infty}$ functions, and we extend them by continuity to the closed interval $0 \leq s \leq L$.

Lemma 1. The extended functions $r, \zeta$ possess right-hand and left-hand derivatives of all orders at $s=0$ and $s=L$ respectively. They have the particular values

$$
\begin{gathered}
r(0)=r(L)=0 ;(d r / d s)(0)=-(d r / d s)(L)=1 ; \\
\zeta(0)=-\zeta(L)=(d C / d s)(0) .
\end{gathered}
$$

Proof. Choose a system of normal coordinates determined by an orthonormal frame at 0 . Let $\zeta_{i}(s), i=1, \cdots, n$, be the components of $\zeta$ with respect to this frame, and $c_{i}(s)$ be the values of the coordinate functions on $C$. Then

Communicated November 12, 1972, and, in revised form, August 1, 1973. 


$$
c_{i}(s)=r(s) \zeta_{i}(s), \quad 0<s<L,
$$

where the $C^{\infty}$ functions $c_{i}$ can be regarded as periodic functions of period $L$.

Because $c_{i}(0)=0$, we can express $c_{i}=s A_{i}$ where the $A_{i}$ are $C^{\infty}$ functions. Consequently, for $s>0$,

$$
\begin{gathered}
r=\left(\sum_{j}{c_{j}}^{2}\right)^{\frac{1}{2}}=s\left(\sum_{j} A_{j}{ }^{2}\right)^{\frac{1}{2}}, \\
\zeta_{i}=A_{i} /\left(\sum_{j} A_{j}{ }^{2}\right)^{\frac{1}{2}}, \quad i, j=1, \cdots, n .
\end{gathered}
$$

The statements in the lemma about the value $s=0$ follow from these formulas and the relations

$$
\left(d c_{i} / d s\right)(0)=A_{i}(0), \quad \sum_{j}\left(\left(d c_{j} / d s\right)(0)\right)^{2}=1 .
$$

Similar arguments can be used to justify the statements about $s=L$. q.e.d.

Let $\Omega$ denote the set of points $(y, s)$ in $R^{2}$ (throughout this paper $R^{i}$ denotes the $i$-dimensional space of real numbers) such that $0 \leq y \leq r(s), 0 \leq s \leq L$, and define $f: \Omega \rightarrow M$ by

$$
f(y, s)=\exp _{0} y \zeta(s)
$$

We call $(\Omega, f)$ the shell on $C$ with vertex 0 . The curve $s \rightarrow \zeta(s)$ which lies on the unit sphere in $T_{0} M$ is called the indicatrix of the shell. Denote by $\Omega_{\varepsilon}$ the

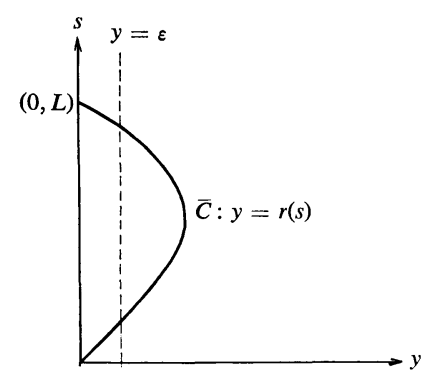

subset of $\Omega$ where $y \geq \varepsilon>0$. Since from Lemma $1, d r / d s$ is continuous and $(d r / d s)(0)=1$, the function $r(s)$ is strictly monotonic increasing on some interval $0 \leq s \leq \alpha$. Similarly, $r(s)$ is strictly monotonic decreasing on some interval $\beta \leq s \leq L$. Now for $s \in[\alpha, \beta], r(s)>0$ and so $r(s)$ has a positive minimum $b$ on $[\alpha, \beta]$. Consider any $\varepsilon$ such that $0<\varepsilon<\min \{r(\alpha), r(\beta), b\}$. The equation $r(s)=\varepsilon$ will have just two solutions for $s \in[0, L]$, thatis, for $\varepsilon$ sufficiently small the line $y=\varepsilon$ will meet the boundary of $\Omega$ in just two points. Essentially our method is to apply the Gauss-Bonnet theorem to the induced 
metric on $\left(\Omega_{\varepsilon}, f\right)$, and then to let $\varepsilon \rightarrow 0$. The key result is Theorem 1 . Unfortunately there are some technical difficulties due to the singularities of $f$.

We will make use of the structure equations for a riemannian $n$-manifold expressed in polar coordinates. Choose an orthonormal frame at 0 , and let $u^{1}, \cdots, u^{n}$ denote the normal coordinates determined by the frame. Extend the frame to a moving frame $X_{1}, \cdots, X_{n}$ on the normal neighborhood by parallel translation along the geodesics through 0 . Denote the dual moving coframe by $\theta^{1}, \cdots, \theta^{n}$, and let $\theta_{j}^{i}=-\theta_{i}^{j}$ be the components of the connection form with respect to these frames.

Define the mapping $F: R^{n+1} \rightarrow M$ by $u^{i}\left(F\left(t, a^{1}, \cdots, a^{n}\right)\right)=t a^{i}, i=1, \cdots, n$. It is shown in $[10$, p. 27] that

$$
F^{*} \theta^{i}=a^{i} d t+\beta^{i}, \quad F^{*} \theta_{j}^{i}=\beta_{j}^{i},
$$

where the forms $\beta^{i}, \beta_{j}^{i}$ do not involve $d t$. These forms are zero for $t=0$, and satisfy the differential equations

$$
\begin{gathered}
\partial \beta^{i} / \partial t=d a^{i}+a^{j} \beta_{j}^{i}, \\
\partial \beta_{j}^{i} / \partial t=\left(R_{j k l}^{i} \circ F\right) a^{k} \beta^{l},
\end{gathered}
$$

where $R_{j k l}^{i}$ are the components of the curvature tensor with respect to the moving frames. For our purposes it is useful to note that

$$
\beta^{i}=t\left(\lambda_{j}^{i} \circ F\right) d a^{j}, \quad a^{j}\left(\lambda_{j}^{i} \circ F\right)=a^{i},
$$

where the $\lambda_{j}^{i}$ are determined by $\theta^{i}=\lambda_{j}^{i} d u^{j}$. Both equalities follow from the first relation in (1).

We denote the components of the vector $\zeta(s)$ with respect to the frame $X_{1}$, $\cdots, X_{n}$ by $\zeta_{i}(s), i=1, \cdots, n$. The mapping $f$ is expressed in terms of the normal coordinates $u^{1}, \cdots, u^{n}$ by $u^{i}(f(y, s))=y \zeta_{i}(s)$. Consequently, at any point $(y, s)$,

$$
f_{*} \frac{\partial}{\partial y}=\zeta_{i} \frac{\partial}{\partial u^{i}}, \quad f_{*} \frac{\partial}{\partial s}=y \frac{d \zeta_{i}}{d s} \frac{\partial}{\partial u^{i}}
$$

Because $\zeta$ is a unit vector, it follows that the vectors $f_{*} \partial / \partial y, f_{*} \partial / \partial s$ are linearly dependent iff $f_{*} \partial / \partial s=0$. Therefore $f$ is an immersion except for points on the lines $y=0$ or $s=\alpha$ where $\alpha$ is any number such that $(d \zeta / d s)(\alpha)=0$. The latter singularities correspond to the points $C(\alpha)$ at which the curve $C$ is tangent to the geodesic from 0 .

In order to use the structure equations we introduce the function $\psi: R^{2} \rightarrow$ $R^{n+1}$ defined by

$$
(y, s) \rightarrow\left(y, \zeta_{1}(s), \cdots, \zeta_{n}(s)\right)
$$


Consequently $f=F \circ \psi$. We define $C^{\infty}$ functions $\omega_{i}, \omega_{j i}=-\omega_{i j}$ in $R^{2}$ by

$$
\psi^{*} \beta^{i}=\omega_{i} d s, \quad \psi^{*} \beta_{j}^{i}=\omega_{j i} d s,
$$

and note that the relations (4) imply that

$$
\omega_{i}=y\left(\lambda_{j}^{i} \circ f\right) d \zeta_{j} / d s, \quad \zeta_{j}\left(\lambda_{j}^{i} \circ f\right)=\zeta_{i} .
$$

It follows from (5) and (7) that, at any point $(y, s)$,

$$
f_{*} \frac{\partial}{\partial y}=\zeta_{j} X_{j}, \quad f_{*} \frac{\partial}{\partial s}=\omega_{j} X_{j} .
$$

The functions $\omega_{i}, \omega_{i j}$ are zero for $y=0$ and satisfy differential equations which are consequences of the structure equations (2) and (3). The equations are

$$
\begin{gathered}
\partial \omega_{i} / \partial y=d \zeta_{i} / d s+\zeta_{j} \omega_{j i} \\
-\partial \omega_{j i} / \partial y=R_{j i k l} \zeta_{k} \omega_{l} .
\end{gathered}
$$

We make one immediate deduction. It follows from (9) that $(\partial / \partial y) \zeta_{i} \omega_{i}=0$. Therefore, because $\zeta_{i} \omega_{i}$ is zero for $y=0$, we can deduce that

$$
\zeta_{i} \omega_{i}=0
$$

The riemannian metric on $M$ induces a metric $\sum_{i}\left(f^{*} \theta_{i}\right)^{2}$ in $R^{2}$. (1), (6), (11) can be used to show that this metric is

$$
d y^{2}+h^{2} d s^{2} \quad \text { where } \quad h=\left(\sum_{i}{\omega_{i}{ }^{2}}^{\frac{1}{2}} .\right.
$$

It is positive definite except at the singularities of $f$, which are therefore the zeros of $h$. The Gaussian curvature $K$ is defined at the nonsingular points and it is easy to check that

$$
K=-\left(\partial^{2} h / \partial y^{2}\right) / h
$$

In fact the integrand $K d A$, where $d A$ is the area element hdyds, still makes sense at the singularities of $f$ on $\Omega_{\varepsilon}$. This will follow from Lemma 4 .

We will need to compare $K$ with the sectional curvature of $M$. We denote by $K_{M}$ the sectional curvature of the plane section spanned by $f_{*} \partial / \partial y, f_{*} \partial / \partial s$. This function is defined at the nonsingular points of $f$, and it follows from (8) that

$$
K_{M}=-R_{j i k l} \zeta_{j} \omega_{i} \zeta_{k} \omega_{l} / h^{2} .
$$

Consequently we obtain from (9) and (10) that 


$$
\partial^{2} \omega_{i} / \partial y^{2}=R_{j i k l} \zeta_{j} \zeta_{k} \omega_{l}
$$

and therefore

$$
K_{M}=-\omega_{i}\left(\partial^{2} \omega_{i} / \partial y^{2}\right) / h^{2} .
$$

In fact, the integrand $K_{M} d A$ still makes sense at the singularities of $f$ on $\Omega$. This will follow from the next lemma. But before we give the proof we introduce a convenient notation. A sequence of functions such as $\omega_{1}, \cdots, \omega_{n}$ will be regarded as the components of a function $\omega$ with values in $R^{n}$, and we will make use of the standard norm and scalar product on $R^{n}$. Thus, for example,

$$
h=\|\omega\|, \quad K_{M}=-\left\langle\omega, \partial^{2} \omega / \partial y^{2}\right\rangle / h^{2} .
$$

Lemma 2. The function $\Lambda_{M}$ defined by

$$
\Lambda_{M}=-\left\langle\omega, \partial^{2} \omega / \partial y^{2}\right\rangle / h, \quad \text { where } h \neq 0 ; \Lambda_{M}=0 \text { otherwise, }
$$

is continuous on $\Omega$.

Proof. $\Lambda_{M}$ is obviously continuous where $h \neq 0$, and at these points

$$
\left|\Lambda_{M}\right| \leq\left\|\partial^{2} \omega / \partial y^{2}\right\|
$$

Clearly this inequality holds at all points of $\Omega$. (13) shows that the functions $\partial^{2} \omega^{i} / \partial y^{2}$ are zero where $h=0$. Consequently the continuity of $\Lambda_{M}$ at these points follows from the inequality and the continuity of the functions $\partial^{2} \omega^{i} / \partial y^{2}$.

The next two lemmas deal with properties of the function $h$.

Lemma 3. The function $\partial h / \partial y$ is continuous on $\Omega$. It is equal to $\|d \zeta / d s\|$ on the line $y=0$ and is zero at the other points where $h=0$.

Proof. The function $h$ is $C^{\infty}$ where it is nonzero and its partial derivative

$$
\partial h / \partial y=\langle\omega, \partial \omega / \partial y\rangle / h .
$$

We will calculate this derivative at the points where $h=0$. According to the first equality in (7), $\omega_{i}=y \mu_{i}$ where $\mu_{i}=\left(\lambda_{j}^{i} \circ f\right) d \zeta_{j} / d s$. Consequently, for $y \geq 0, h=y\|\mu\|$. Now $\lambda_{j}^{i}(0)=\delta_{j}^{i}$, and therefore $\mu(0, s)=d \zeta / d s$. It follows that for points on the line $y=0$ the right-hand derivative

$$
\partial h / \partial y=\|\mu(0, s)\|=\|d \zeta / d s\| \text {. }
$$

The other points where $h=0$ lie on the lines $s=\alpha$ where $\alpha$ is any number such that $(d \zeta / d s)(\alpha)=0$. At these points $\partial h / \partial y=0$.

It follows from the formula $h=y\|\mu\|$ that $\partial h / \partial y$ is continuous at the points on the line $y=0$ where $\mu \neq 0$. The other points at which $h=0$ are the points $(a, \alpha)$ such that $(d \zeta / d s)(\alpha)=0$. To demontrate continuity at these points we use the inequality 


$$
|\partial h / \partial y| \leq\|\partial \omega / \partial y\| .
$$

For $h \neq 0$ this inequality is a consequence of (16), and it is easy to check that it also holds where $h=0$. The functions $\partial \omega_{i} / \partial y$ are of course continuous, and are zero at $(a, \alpha)$. Therefore it follows from (17) that $\partial h / \partial y$ is continuous at $(a, \alpha)$.

Lemma 4. The function $\partial^{2} h / \partial y^{2}$ is continuous on $\Omega_{\varepsilon}$.

Proof. It is convenient to put

$$
\Lambda=-\partial^{2} h / \partial y^{2} .
$$

We find from (16) that at points where $h \neq 0$

$$
\Lambda=\Lambda_{M}-h^{-3}\left(\|\omega\|^{2}\|\partial \omega / \partial y\|^{2}-\langle\omega, \partial \omega / \partial y\rangle^{2}\right) .
$$

The points of $\Omega_{\varepsilon}$ at which $h=0$ lie on the lines $s=\alpha$ where $\alpha$ is such that $(d \zeta / d s)(\alpha)=0$. Consequently $\Lambda=0$ at these points.

The continuity of $\Lambda$ at points where $h \neq 0$ is obvious from (19). According to Lemma 2 the continuity of $A$ at the zeros of $h$ will follow from that of $\Lambda_{M}-\Lambda$. To establish this we obtain from the first equality in (7)

$$
\frac{\partial \omega_{i}}{\partial y}=\frac{1}{y}\left(\omega_{i}+y\left(\frac{\partial \lambda_{j}^{i}}{\partial u^{k}} \circ f\right) \zeta_{k} \mu_{r}^{j} \omega_{r}\right)
$$

where $\left[\mu_{i}^{j}\right]$ is the matrix inverse to $\left[\lambda_{i}^{j} \circ f\right]$. The right-hand side is linear in the functions $\omega_{i}$ with coefficients which are bounded on $\Omega_{\varepsilon}$. Consequently an inequality

$$
\|\partial \omega / \partial y\| \leq A\|\omega\|=A h
$$

is valid on $\Omega_{\varepsilon}$ where $A$ is some constant depending on $\varepsilon$. Therefore using (19), we find

$$
0 \leq \Lambda_{M}-\Lambda \leq\|\partial \omega / \partial y\|^{2} / h \leq A^{2} h
$$

and this inequality implies that $\Lambda_{M}-\Lambda$ is continuous at the zeros of $h$.

We have to consider also the geodesic curvature $\kappa$ of the curve $\bar{C}: s \rightarrow(r(s), s)$ in $(\Omega, f)$. This curvature is defined at the points where $f$ is an immersion, and it is easy to check that

$$
\kappa=\partial h / \partial y-\left(d^{2} r / d s^{2}\right) / h .
$$

Let $\kappa_{M}$ denote the geodesic curvature of the curve $C$ in $M, t$ the unit tangent vector of $C$, and $\gamma$ the second fundamental form of $(\Omega, f)$. Then from the wellknown formula 


$$
\kappa_{M}^{2}=\kappa^{2}+\text { the square of the length of } \gamma,
$$

it is clear that $\left|\kappa_{M}\right| \geq|\kappa|$. Our aim is to extend $\kappa$ to an integrable function, defined almost everywhere on the interval $[0, L]$ such that the inequality continues to hold.

It is convenient to define $k(s)=h(r(s), s), \phi=\omega(r(s), s)$ so that $\kappa=\|\phi\|$. As the parameter $s$ is the arc length along $C$ it follows that

$$
k^{2}+(d r / d s)^{2}=1 .
$$

The following lemma establishes properties of the function $k$.

Lemma 5. (i) $k$ is absolutely continuous on $[0, L]$.

(ii) $k$ is differentiable at the points where it is nonzero. It is differentiable at a zero $s=\alpha$ iff $(d \phi / d s)(\alpha)=0$.

Proof. Because $\phi$ is a $C^{\infty}$ function, there is a constant $B$ such that $\|d \phi / d s\| \leq B$ on $[0, L]$. We obtain, using the mean value theorem,

$$
|k(b)-k(a)|=|\|\phi(b)\|-\|\phi(a)\|| \leq\|\phi(b)-\phi(a)\| \leq B(b-a),
$$

where $0 \leq a<b \leq L$. This Lipschitz condition implies that $k$ is absolutely continuous.

The first statement in (ii) is obvious. To prove the second we suppose that $\phi(\alpha)=0$ but $(d \phi / d s)(\alpha) \neq 0$. Then $\phi=(s-\alpha) \psi$ where $\psi$ is a $C^{\infty}$ function and $\psi(\alpha) \neq 0$. Consequently $k=|s-\alpha|\|\psi\|$ is not differentiable at $s=\alpha$. On the other hand if $(d \phi / d s)(\alpha)=0$, then $\phi=(s-\alpha)^{2} \lambda$ where $\lambda$ is a $C^{\infty}$ function. Consequently $k=(s-\alpha)^{2}\|\lambda\|$ has a zero derivative at $s=\alpha$.

We will show that the geodesic curvature can be extended by using the function $\theta$ defined on $[0, L]$ by

$$
\sin \theta=d r / d s, \quad-\frac{1}{2} \pi \leq \theta \leq \frac{1}{2} \pi .
$$

It follows from (22) that $\cos \theta=k$. In the induced metric on $(\Omega, f), \theta$ is the angle between the tangent vector to $\bar{C}$ and the $s$-axis. The required properties of $\theta$ are established in

Lemma 6. The function $\theta$ is absolutely continuous on $[0, L]$. It is differentiable at a point $\alpha$ iff $k$ is differentiable at $\alpha$.

Proof. The function $\sin ^{-1}$ is uniformly continuous on the closed interval $[-1,1]$, and so there exists a number $\lambda>0$ such that $\left.\mid \sin ^{-1} \alpha-\sin ^{-1} \beta\right]<\frac{1}{2} \pi$ when $|\alpha-\beta|<\lambda,-1 \leq \alpha, \beta \leq 1$. The function $r$ is $C^{\infty}$ on $[0, L]$, and so there exists a number $B>0$ such that $\left|d^{2} r / d s^{2}\right|<B$ on $[0, L]$. Let $s_{1}$, $s_{2}$ be such that $0 \leq s_{1}, s_{2} \leq L,\left|s_{1}-s_{2}\right|<\lambda / B$. We will write $\theta_{i}, r_{i}^{\prime}, k_{i}$ for the values of the functions $\theta, r^{\prime}=d r / d s, k$ at the points $s_{i}, i=1,2$. From the definition of $\theta$ we obtain

$$
\sin \left(\theta_{2}-\theta_{1}\right)=r_{2}^{\prime} k_{1}-r_{1}^{\prime} k_{2}=k_{1}\left(r_{2}^{\prime}-r_{1}^{\prime}\right)-r_{1}^{\prime}\left(k_{2}-k_{1}\right),
$$


and therefore

$$
\left|\sin \left(\theta_{2}-\theta_{1}\right)\right| \leq\left|r_{2}^{\prime}-r_{1}^{\prime}\right|+\left|k_{2}-k_{1}\right| \text {. }
$$

It follows from the mean value theorem that $\left|\sin \theta_{2}-\sin \theta_{1}\right|<\lambda$. Consequently $\left|\theta_{2}-\theta_{1}\right|<\frac{1}{2} \pi$, and so we may use the inequality $\left|\theta_{2}-\theta_{1}\right|<\frac{1}{2} \pi\left|\sin \left(\theta_{2}-\theta_{1}\right)\right|$ to conclude that

$$
\left|\theta_{2}-\theta_{1}\right|<\frac{1}{2} \pi\left(\left|r_{2}^{\prime}-r_{1}^{\prime}\right|+\left|k_{2}-k_{1}\right|\right) .
$$

The absolute continuity of $\theta$ now follows from that of the function $k$ (Lemma 5) and the $C^{\infty}$ function $d r / d s$.

To investigate the differentiability of $\theta$ we consider first the points where $k \neq 0$. At these points $\cos \theta \neq 0$, and the formula $\sin \theta=d r / d s$ gives

$$
d \theta / d s=\left(d^{2} r / d s^{2}\right) / k .
$$

On the other hand, in a neighborhood of a zero of $k$ the function $\sin \theta \neq 0$, and the formula $\cos \theta=k$ gives

$$
d \theta / d s=-(d k / d s) /(d r / d s)
$$

iff $d k / d s$ exists.

Since an absolutely continuous function is differentiable almost everywhere [4, p. 205], it follows from Lemma 3, Lemma 6 and the formulas (21) and (23) that the definition

$$
\kappa=\partial h / \partial y-d \theta / d s
$$

extends the domain of $\kappa$ to $[0, L]$ except for a set of Lebesgue measure zero. Further, the proofs of the lemmas show that $\kappa=0$ where $k=0$. Consequently the inequality $\left|\kappa_{M}\right| \geq|\kappa|$ holds almost everywhere on $[0, L]$.

We are now able to prove the key theorem. The statement of this theorem makes use of the meanings which we have attached to curvatures at the singularities of $f$.

Theorem 1. Let 0 be a point on a closed $C^{\infty}$ curve $C$ imbedded in a riemannian n-manifold $M$, and suppose that $C$ lies in a normal neighborhood of 0 . Construct the shell $(\Omega, f)$ on $C$ with vertex 0 . Let $K$ be the Gaussian curvature of the induced metric on $(\Omega, f)$, and use $d A$ for its area measure. Denote by $\kappa$ the geodesic curvature of $C$ considered as a curve in $(\Omega, f)$, and let $s$ be its arc length. Then

$$
\int_{0}^{L} \kappa d s=\pi+l-\int_{\Omega} K d A
$$


where $L$ is the length of $C$, and $l$ is the length of the indicatrix of the shell.

Proof. Let $s_{1}, L-s_{2}$ be the values of $s$ at which the line $y=\varepsilon$ meets the curve $y=r(s)$. By definition

$$
\begin{aligned}
\int_{\Omega} K d A & =\lim _{\varepsilon \rightarrow 0} \int_{\Omega_{\varepsilon}}-\frac{\partial^{2} h}{\partial y^{2}} d y d s \\
& =\lim _{\varepsilon \rightarrow 0}\left(-\int_{s_{1}}^{L-s_{2}} \frac{\partial h}{\partial y}(r(s), s) d s+\int_{s_{1}}^{L-s_{2}} \frac{\partial h}{\partial y}(\varepsilon, s) d s\right) .
\end{aligned}
$$

It follows from Lemma 3 that

$$
\int_{\Omega} K d A=-\int_{0}^{L} \frac{\partial h}{\partial y}(r(s), s) d s+l .
$$

The proof is completed by substituting for $\partial h / \partial y$ from the definition (24). For, according to Lemma $6, \theta$ is absolutely continuous and therefore [4, p. 207]

$$
\int_{0}^{L} \frac{d \theta}{d s} d s=\theta(L)-\theta(0)
$$

Lemma 1 shows that the right-hand side is equal to $-\pi$.

\section{An inequality for the total absolute curvature}

Theorem 2. Let $M$ be a complete simply connected riemannian manifold with a nonpositive sectional curvature function $K_{M}$. Then the geodesic curvature $\kappa_{M}$ of any closed $C^{\infty}$ curve $C$ imbedded in $M$ satisfies the inequality

$$
\int_{C}\left|\kappa_{M}\right| d s \geq 2 \pi-\int_{\Omega} K_{M} d A
$$

where $(\Omega, f)$ is any shell on $C$.

Proof. It is well known that such a manifold $M$ is a normal neighborhood of each of its points so that the shell $(\Omega, f)$ is defined. According to Lemma 1 the indicatrix of the shell joins a pair of antipodal points on a unit sphere and therefore its length $l \geq \pi$. Consequently as $\left|\kappa_{M}\right| \geq|\kappa|$ we obtain, using Theorem 1 ,

$$
\int_{C}\left|\kappa_{M}\right| d s \geq \int_{0}^{L}|\kappa| d s \geq \int_{0}^{L} \kappa d s=\pi+l-\int_{\Omega} K d A \geq 2 \pi-\int_{\Omega} K d A .
$$

The proof is completed by $\left(K_{M}-K\right) d A=\left(\Lambda_{M}-\Lambda\right) d y d s \geq 0$, which follows from (12), (14), (15), (18), (20) (on $M$ wherever $K$ and $K_{M}$ are defined the formula $K_{M}-K \geq 0$ is due to J. L. Synge [8]). 
It should be remarked that by a completely different method J. Szenthe [9] has shown that $\int_{C}\left|\kappa_{M}\right| d s \geq 2 \pi$, which is obviously weaker than our inequality (25).

For $K_{M} \neq 0$, from (25) we prove immediately the following conjecture of N. H. Kuiper :

Corollary. The total absolute curvature of any closed $C^{\infty}$ curve imbedded in a complete simply connected riemannian manifold with negative sectional curvature is greater than $2 \pi$.

We can obtain more precise results in the case when $M$ has constant sectional curvature. But we will need some information about imbedded closed curves in the hyperbolic and euclidean planes. As in euclidean space we will say that a subset $S$ of hyperbolic space is convex if $\{P, Q\} \subset S$ implies that the geodesic segment joining $P$ to $Q$ also lies in $S$. An imbedded closed curve $C$ in the hyperbolic plane is said to be convex if it is the boundary of a convex subset of the plane. In our case $C$ will be of class $C^{\infty}$, and then an equivalent condition is that $C$ lie entirely on one side of each tangent geodesic.

Theorem 3. Let $M$ be a hyperbolic (resp. euclidean) space, and denote its sectional curvature by $\nu$. Then the geodesic curvature $\kappa_{M}$ of any closed $C^{\infty}$ curve $C$ imbedded in $M$ satisfies the inequality

$$
\int_{C}\left|\kappa_{M}\right| d s \geq 2 \pi-\nu A
$$

where $A$ is the area of any shell on $C$. If the equality occurs for some shell on $C$, then $C$ is imbedded as a closed convex curve in a hyperbolic (resp. euclidean) plane in $M$.

Proof. The first statement is a special case of Theorem 2. To prove the second statement we suppose that the equality occurs for a shell $(\Omega, f)$ on $C$ with vertex 0 . According to the proof of Theorem 2 the indicatrix of this shell has length $\pi$ and is therefore a great semi-circle on the unit sphere in $T_{0} M$. Consequently $C$ lies in the totally geodesic surface $S$ through 0 tangent to the plane of this great semi-circle. The surface $S$ with its induced metric is a hyperbolic (resp. euclidean) plane in $M$, and the geodesic curvature of $C$, considered as a curve in $S$, is equal to $\kappa_{M}$. We will need

Lemma 7. $\kappa_{M}$ has a constant sign.

Proof. It is clear that $\kappa_{M}=\kappa$ at the nonsingular points of $f$, that is, where $h \neq 0$. Suppose that $\kappa_{M}(\alpha)>0$. Then $\kappa_{M}>0$ on some open interval $I$ containing $\alpha$. The function $h$ is not everywhere zero on $I$, because that implies the restriction of $C$ to $I$ is a geodesic and so $\kappa_{M}(\alpha)=0$. Therefore $\kappa=\kappa_{M}>0$ on some subinterval of $I$. Similarly, the supposition $\kappa_{M}(\alpha)<0$ implies that $\kappa<0$ on some interval of values of $s$. Consequently, unless $\kappa_{M}$ has a constant sign, there is a strict inequality 


$$
\int_{0}^{L}|\kappa| d s>\int_{0}^{L} \kappa d s
$$

and so, from the proof of Theorem 2, a strict inequality in the statement of Theorem 3.

It follows from Lemma 7 that $C$ is convex. This fact is well known in the euclidean case $[1$, p. 21], and we prove it for the hyperbolic case in the appendix (Theorem 5).

Theorem 3 is an extension of a well-known theorem of Fenchel [3]. The following theorem is an extension of the theorems of Fary [2] and Milnor [5].

Theorem 4. Let $M$ be a hyperbolic or euclidean space of dimension three, and denote its sectional curvature by $\nu$. Let $\kappa_{M}$ be the geodesic curvature of a $C^{\infty}$ knot $C$ in $M$. Then for some shell on $C$

$$
\int_{C}\left|\kappa_{M}\right| d s \geq 4 \pi-\nu A
$$

where $A$ is the area of the shell.

Proof. Let $D$ denote a closed imbedded $C^{\infty}$ curve in $M$ such that there is no shell on $D$ which satisfies the stated inequality. Therefore, for any point 0 on $D$,

$$
\int_{D}\left|\kappa_{M}\right| d s<4 \pi-\nu A
$$

where $A$ is the area of the shell on $D$ with vertex 0 . On the other hand, the proof of Theorem 2 contains the inequality

$$
\int_{D}\left|\kappa_{M}\right| d s \geq \pi+l-\nu A
$$

where $l$ is the length of the indicatrix of the shell. Consequently $l<3 \pi$. Our aim is to show that this condition implies that $D$ is unknotted. We will prove first that the condition implies there is a plane through 0 (hyperbolic or euclidean respectively) which meets $D$ in just one other point and is transversal to $D$ at both points of intersection. Then Theorem 6, which is proved in the appendix, shows that $D$ is unknotted.

Consider an oriented great circle $G$ on the unit sphere $\Sigma$ in $T_{0} M . G$ lies in a plane $\gamma$ in $T_{0} M$ and gives an orientation to $\gamma$. Choose an orientation for $T_{0} M$. This orientation is determined by the orientation of $\gamma$ and a unit vector normal to $\gamma$. The measure of a set of oriented great circles on $\Sigma$ is, by definition, the area measure of the corresponding unit normals considered as points of $\Sigma$. We attach to each great circle $G$ the number $n(G)$ of its points of intersection with the indicatrix of the shell. Applying Crofton's theorem [1, p. 33] we find that 


$$
\int n(G) d G=4 \pi l<12 \pi
$$

It is clear that $n(G) \geq 1$, which, together with (26) and the following Lemma 8, implies that the set of great circles on $\Sigma$ with $n(G)=1$ has strictly positive measure because otherwise

$$
\int n(G) d G \geq \int 3 d G=12 \pi
$$

Lemma 8. The measure of the set of great circles on $\Sigma$ with $n(G)=2$ is zero.

Proof. The indicatrix $\zeta: s \rightarrow \zeta(s)$ joins antipodal points $\boldsymbol{P}, \boldsymbol{Q}$ on $\Sigma$. Any great circle on $\Sigma$ meeting $\zeta$ in just two points either goes through $\boldsymbol{P}$ and $\boldsymbol{Q}$ or is tangent to $\zeta$ for some $s, 0<s<L$ ("tangent to $\zeta$ " is to include going through a singular point where $d \zeta / d s=0$ ).

Consider the set $S_{1}$ of oriented great circles on $\Sigma$ through $\boldsymbol{P}$ and $\boldsymbol{Q}$. Since their normals lie on a great circle on $\Sigma$, obviously $S_{1}$ has measure zero.

Consider the set $S_{2}$ of great circles on $\Sigma$ tangent to $\zeta$ for some $s, 0<s<L$. If $l=l_{i} x_{i}(0)$ is the normal to the plane of such a great circle, then

$$
l_{i} \zeta_{i}(s)=0, \quad l_{i} d \zeta_{i} / d s=0 .
$$

We will show that $S_{2}$ has measure zero by using Sard's theorem [6]. Consider the projection $\Sigma \times I \rightarrow \Sigma$ where $I$ is the open interval $(0, L)$. Let $\chi$ be the restriction of this projection to the 2-dimensional submanifold $N$ of points $(l, s)$ such that $l_{i} \zeta_{i}=0$. A point $p=(l, s) \in N$ is critical for $\chi$ iff $\partial / \partial s$ is tangent to $N$ at $p$. This is so iff $l_{i} d \zeta_{i} / d s=0$. Consequently the set of critical values of $\chi$ is the set of unit vectors $l$ satisfying (27). Sard's theorem implies that this set (and hence $S_{2}$ ) has measure zero.

The set of great circles with $n(G)=2$ is contained in the union $S_{1} \cup S_{2}$ and therefore has measure zero. q.e.d.

Each oriented great circle $G$ determines an oriented plane $\sigma$ in $M$ (hyperbolic or euclidean respectively) whose tangent plane at 0 contains $G$. The planes corresponding to the great circles with $n(G)=1$ are transversal to $D$ at 0 , and meet $D$ in just one other point. We have to show that some of these planes are transversal to $D$ at both points of intersection. Let $P$ denote a general point on $D$ distinct from 0 , and $\sigma$ an oriented plane containing $P$ and 0 . Consider those planes which are not transversal to $D$ at $P$.

Lemma 9. As $P$ varies on $D$, the union of the corresponding great circles on $\Sigma$ has measure zero.

Proof. We will show that this union is the set $S_{2}$ introduced in the proof of Lemma 8. Consider an oriented plane $\sigma$ containing $P$ and 0 , and suppose 
that its normal at 0 is $l_{i} x_{i}(0)$. Then its normal at $P$ is $l_{i} x_{i}(P)$. The plane $\sigma$ contains the geodesic $O P$ and therefore

$$
l_{i} \zeta_{i}(s)=0 .
$$

It follows from (8) that $\sigma$ is not transversal to $D$ iff $l_{i} \omega_{i}(r(s), s)=0$. For spaces of constant nonpositive sectional curvature $\nu$ the functions $\omega_{i}$ are proportional to $d \zeta_{i} / d s$. To show this we will integrate (13) which simplifies to

$$
\partial^{2} \omega_{i} / \partial y^{2}=-\nu \omega_{i}
$$

Imposing the initial conditions $\omega_{i}=0$, and $\partial \omega_{i} / \partial y=d \zeta_{i} / d s$ when $y=0$, we find

$$
\begin{array}{rlrl}
\omega_{i} & =\frac{1}{\sqrt{-\nu}} \sinh (\sqrt{-\nu} y) \frac{d \zeta_{i}}{d s}, & & \nu<0, \\
\omega_{i}=y d \zeta_{i} / d s, & \nu=0 .
\end{array}
$$

Consequently $\sigma$ is not transversal to $D$ iff

$$
l_{i} d \zeta_{i} / d s=0 .
$$

As (28) and (29) are the same as (27), it follows that the set of great circles which we are considering is the set $S_{2}$. q.e.d.

Consequently by Lemma 9, almost all great circles on $\Sigma$ with $n(G)=1$ determine planes which are transversal to $D$ at both points of intersection.

\section{Appendix}

In this appendix we prove two theorems which have been used in our previous work.

Theorem 5. Let $\mathrm{H}$ be the hyperbolic plane of constant sectional curvature -1 , and $C$ be a closed $C^{\infty}$ curve imbedded in $H$. Then $C$ is convex iff it can be oriented so that its geodesic curvature $\kappa \geq 0$.

Proof. Let $(x, y)$ be the standard coordinates on $R^{2}$. We will take as our model of $H$ the subset of $R^{2}$ where $x>0$, together with the metric

$$
\frac{(d x)^{2}+(d y)^{2}}{x^{2}} \text {. }
$$

The sign of the geodesic curvature of a curve in $H$ depends on a choice of an orientation for $H$. We choose the orientation defined by the chart $(x, y)$. Then it is easy to derive the formula

$$
\kappa=\left(x^{\prime} y^{\prime \prime}-y^{\prime} x^{\prime \prime}-x y^{\prime}\right) / x^{2},
$$


where the primes denote differentiation with respect to the arc length $s$. It follows at once that the lines $y=$ constant are geodesics in $H$, and our proofs will make use of this fact. If a curve $C$ is tangent to such a geodesic, then in a neighborhood of the point of contact we can use the coordinate $x$ as a parameter on $C$. It is not difficult to verify the formula

$$
\kappa=x^{2} \frac{d}{d x}\left(\frac{y^{\prime}}{x^{2}}\right) \text {. }
$$

We are now ready to begin the proof of the theorem. To show that the stated condition is necessary we suppose that $C$ is convex, but that it cannot be oriented to have its geodesic curvature $\kappa \geq 0$. Therefore there are points on $C$ at which $\kappa>0$, and also points where $\kappa<0$. Consider a point where $\kappa>0$, and choose the arc length parameter so that this point has parameter value zero. Let $\alpha$ be the supremum of those values of $s$ for which $\kappa(t) \geq 0,0 \leq t \leq s$. Then $\kappa(\alpha)=0$, and in any neighborhood of $\alpha$ there are values of $s$ for which $\kappa(s)<0$.

Let $P$ denote the point on $C$ of parameter $\alpha$. We can use a proper isometry of $H$ to move $C$ so that it becomes tangent at $P$ to the $x$-axis with $x^{\prime}>0$ at $P$. The curve $C$ remains convex, and its curvature is unaltered. Because $\kappa(s) \geq 0$ for $0 \leq s \leq \alpha$, the formula (31) shows that some part of $C$ lies above the $x$-axis. Therefore, because $C$ is convex and the $x$-axis is a geodesic, $C$ lies entirely above the $x$-axis. Of course, if $Q$ is any other point on $C$, we can again move $C$ so that it becomes tangent to the $x$-axis at $Q$ with $x^{\prime}>0$ at $Q$. But if $Q$ is near to $P$, then we need only move $C$ a small amount. Consequently, if $Q$ is near enough to $P$, then $C$ will still lie above the $x$-axis.

We can choose $Q$ so that $\kappa<0$ at $Q$. Because $y^{\prime}=0$ at $Q$, it follows from the formula (30) that $y^{\prime \prime}<0$ at $Q$. This inequality contradicts the previous conclusion that $C$ lies above the $x$-axis. Therefore $C$ can be oriented so that $\kappa \geq 0$.

To prove the converse implication we begin with a technical lemma.

Lemma 10. Let $C$ be a $C^{\infty}$ closed curve imbedded in the hyperbolic plane $H$ with geodesic curvature $\kappa \geq 0$. Suppose that a geodesic $y=$ constant is tangent to $C$ at a point $P$ of arc length parameter $\alpha$, and that $x^{\prime}(\alpha)>0$. Then there exist numbers $a, b$ such that

$$
\begin{gathered}
a<\alpha<b, y(s) \geq y(\alpha) \text { for } a \leq s \leq b, \\
y(a)>y(\alpha), \quad y(b)>y(\alpha) .
\end{gathered}
$$

Proof. Let $\left[a^{\prime}, b^{\prime}\right]$ be the largest closed interval containing $\alpha$ on which $\kappa=0$. On this interval the curve $C$ coincides with the geodesic $y=y(\alpha)$. We can choose $a, b$ to satisfy the conditions $a<a^{\prime}, b^{\prime}<b$ and $x^{\prime}>0$ on the interval $[a, b]$. The fact that $a, b$ also satisfy the conditions stated in the lemma is an easy consequence of the formula (31). q.e.d. 
To complete the proof of the theorem we suppose that $\kappa \geq 0$ on $C$, but that $C$ is not convex. Consequently there are points of $C$ on both sides of some tangent geodesic. We can move $C$ so that this tangent geodesic becomes the $x$-axis with $x^{\prime}>0$ at the point of contact, $P$. Lemma 10 gives us information about the shape of $C$ near to $P$. But there are also points on $C$ for which the $y$-coordinate is strictly negative. It follows that there is at least one local maximum for $y$ on $C$ at which $x^{\prime}>0$. Lemma 10 implies that such a local maximum cannot exist. Therefore $C$ must be convex.

In the next theorem we will be dealing with hyperbolic or euclidean space $M$ of dimension three. Let $D$ be a closed piecewise $C^{\infty}$ curve imbedded in $M$. A plane (hyperbolic or euclidean respectively), which meets $D$ in just two points and its transversal to $D$ at each point of intersection, will be said to be transversal to $D$. In this definition a plane is transversal to $D$ at a point of intersection of two $C^{\infty}$ arcs if it is transversal to both arcs and also separates them. It is not difficult to see that if a plane $p$ is transversal to $D$, then any plane sufficiently near to $p$ is also transversal to $D$. If $D$ admits a transversal plane through each of its points, then we will say that it has the transversal property.

Theorem 6. Let $C$ be a closed piecewise $C^{\infty}$ curve embedded in hyperbolic or euclidean space $M$ of dimension three. If $C$ has the transversal property, then $C$ is a trivial knot.

Proof. (The idea of the proof is due to T. Poston.) Let $D$ be a closed piecewise $C^{\infty}$ curve embedded in $M$, and suppose that $D$ has the transversal property. We will describe a procedure for splitting $D$ into two new closed curves which also have the transversal property. Choose a point $Q$ on $D$ and a plane $q$ through $Q$ transversal to $D$. Let $R$ denote the second point of intersection. The plane $q$ divides $D$ into two arcs $\Gamma_{1}, \Gamma_{2}$, and we complete these arcs to closed curves $D_{1}, D_{2}$ by adding on the geodesic segment $Q R$.

To show that the new curves $D_{1}, D_{2}$ also have the transversal property we restrict our attention to $D_{1}$. First of all note that if $P$ lies on the segment $Q R$, then a suitable small variation of the plane $q$ will provide a plane through $P$ transversal to $D_{1}$. If $P$ is not on this segment, then there is a plane $p$ through $P$, transversal to $D$, which does not go through $Q$ or $R$. If $p$ meets $D$ again in the arc $\Gamma_{1}$, then it cannot meet the segment $Q R$ and is therefore a transversal plane for $D_{1}$. On the other hand, if $p$ meets $D$ again in the $\operatorname{arc} \Gamma_{2}$, then it must also meet the segment $Q R$. It will meet this segment transversally and so will again be a transversal plane for $D_{1}$.

We now outline the small amount of knot theory which we need. For details we refer to [7]. The procedure we have just described factorizes the knot $D$ into the product of the knots $D_{1}, D_{2}$. Associated with any knot is a nonnegative integer called the genus of the knot. It satisfies the simple relation.

$$
\text { genus } D=\text { genus } D_{1}+\text { genus } D_{2} \text {. }
$$


This relation, together with the fact that a knot is trivial iff it has genus zero, is all that we use.

We will say that the closed curve $D$ satisfies condition $A$ if, for any splitting of $D$ into closed curves $D_{1}, D_{2}$, at least one of the curves is a trivial knot. As a product of a finite number of trivial knots is a trivial knot, Theorem 6 is a consequence of the following lemmas.

Lemma 11. A piecewise $C^{\infty}$ knot $C$ which has the transversal property can be split into a product of knots each satisfying condition $A$.

Proof. Either $C$ itself satisfies conditon $A$ or it can split into a product of knots $C_{1}, C_{2}$ which have the transversal property and which are not trivial knots. The relation (32) shows that $C_{1}$ and $C_{2}$ will each have a genus strictly less than that of $C$. If $C_{1}, C_{2}$ both satisfy condition $A$, then the proof is complete. Otherwise we can continue by splitting $C_{1}$ or $C_{2}$ (or both) into products. This process will terminate after a finite number of steps because the genus of a knot is a nonnegative integer.

Lemma 12. A piecewise $C^{\infty}$ knot $D$ which satisfies condition $A$ is a trivial knot.

Proof. We will say that an $\operatorname{arc} \Gamma$ of $D$ is trivial, if there is a transversal plane through its end points $Q, R$, and the closed curve formed by $\Gamma$ and the geodesic segment $Q R$ is a trivial knot.

We first note that any point $P$ on $D$ lies in the interior of a trival arc. For consider a plane $p$ through $P$ transversal to $D$, and let $P^{\prime}$ denote its second point of intersection with $D$. The plane $p$ divides $D$ into two arcs at least one of which is trivial. Rotate $p$ about $P^{\prime}$ to increase the length of the trivial arc. If the rotation is sufficiently small, the new arc will also be trivial. It will, of course, include $P$ in its interior.

Because $D$ is compact, we can cover $D$ by a finite number of trivial arcs. We can also arrange that the cover is minimal in the sense that no arc is contained in the union of the remaining arcs of the cover. Let $\Gamma_{1}, \cdots, \Gamma_{n}$ be such a minimal cover, and let $Q_{i}, R_{i}$ be the end points of $\Gamma_{i}$. Divide $D$ into two arcs by a transversal plane $q_{1}$ through $Q_{1}, R_{1}$, and construct a new knot $D^{\prime}$ by replacing the arc $\Gamma_{1}$ by the geodesic segment $Q_{1} R_{1}$. The relation (32) shows that if $D^{\prime}$ is a trivial knot, then so is $D$. Apart from the segment $Q_{1} R_{1}$ the knot $D^{\prime}$ lies entirely on one side of $q_{1}$, and we will say that it lies in a half-space $\pi_{1}$.

Now consider the knot $D^{\prime}$. Some of the $\operatorname{arcs} \Gamma_{2}, \cdots, \Gamma_{n}$ will be $\operatorname{arcs}$ of $D^{\prime}$, and the remainder will have just one end point on $\Gamma_{1}$. Let $\Gamma_{\alpha}$ denote an arc with an end point $Q_{\alpha}$ on $\Gamma_{1}$. There is a plane $q_{\alpha}$ through $Q_{\alpha}, R_{\alpha}$ transversal to $D$. The plane $q_{\alpha}$ must intersect the segment $Q_{1} R_{1}$, and we will denote the point of intersection by $Q_{\alpha}^{\prime}$. One of the $\operatorname{arcs} Q_{1} R_{\alpha}$ or $R_{1} R_{\alpha}$ is contained in $\Gamma_{\alpha}$. The union of this arc with the segment $Q_{\alpha}^{\prime} Q_{1}$ or $Q_{\alpha}^{\prime} R_{1}$ respectively, gives an arc $\Gamma_{\alpha}^{\prime}$ of $D^{\prime}$.

It is not difficult to show that $\Gamma_{\alpha}^{\prime}$ is a trivial arc of $D^{\prime}$. In fact, for this pur- 
pose by assuming $Q_{1}$ to be on $\Gamma_{\alpha}$ we have to show that the closed curve $E$ consisting of the segment $Q_{\alpha}^{\prime} Q_{1}$, the part of $\Gamma_{\alpha}$ from $Q_{1}$ to $R_{\alpha}$ and the segment $R_{\alpha} Q_{\alpha}^{\prime}$ is a trivial knot. We are given that $\Gamma_{\alpha}$ is a trivial arc of $D$. This means that the closed curve $F$ consisting of the arc $\Gamma_{\alpha}$ and the segment $R_{\alpha} Q_{\alpha}$ is a trivial knot. Consider the closed curve $G$ consisting of the $\operatorname{arc} \Gamma_{\alpha}$, the segment $R_{\alpha} Q_{\alpha}^{\prime}$ and the segment $Q_{\alpha}^{\prime} Q_{\alpha}$. $G$ is isotopic to $F$ because we can deform the broken geodesic segment $R_{\alpha} Q_{\alpha}^{\prime} Q_{\alpha}$ into the segment $R_{\alpha} Q_{\alpha}$ keeping within the transversal plane $q_{\alpha}$. Since $F$ is a trivial knot, $G$ is also a trivial knot and therefore genus $G=0$. Since the knot $G$ is the product of the knot $E$ and the knot consisting of the arc of $\Gamma_{\alpha}$ from $Q_{\alpha}$ to $Q_{1}$, the segment $Q_{1} Q_{\alpha}^{\prime}$ and the segment $Q_{\alpha}^{\prime} Q_{\alpha}$, the relation (32) implies that genus $E=0$, and hence that $E$ is a trivial knot.

We carry out the above modifications where necessary, and obtain a cover of $D^{\prime}$ consisting of the segment $Q_{1} R_{1}$ and trivial $\operatorname{arcs} \Gamma_{2}^{\prime}, \cdots, \Gamma_{n}^{\prime}$.

We can now construct another new knot $D^{\prime \prime}$ by replacing $\Gamma_{2}^{\prime}$ by a geodesic segment lying in a transversal plane $q_{2}$. As before $D^{\prime \prime}$ lies in a half-space $\pi_{2}$, and if $D^{\prime \prime}$ is trivial then so is $D^{\prime}$. After $n$ such steps we will arrive at a knot $S$ consisting entirely of geodesic segments. $S$ will lie on the boundary of the convex set formed by the intersection of the half-spaces $\pi_{1}, \cdots, \pi_{n}$, and will therefore be a trivial knot. Consequently $D$ is a trivial knot.

\section{References}

[1] S. S. Chern, Curves and surfaces in Euclidean space, Studies in Global Geometry and Analysis, Studies in Math., Vol. 4, Math. Assoc. Amer., 1967, 16-56.

[2] I. Fary, Sur la courbure totale d'une courbe gauche faisant un noeud, Bull. Soc. Math. France 77 (1949) 128-138.

[3] W. Fenchel, Über Krümmung und Windung geschlossener Raumkurven, Math. Ann. 101 (1929) 238-252.

[4] L. M. Graves, The theory of functions of real variables, Second edition, McGrawHill, New York, 1956.

[5] J. Milnor, On the total curvature of knots, Ann. of Math. 52 (1950) 248-257.

[6] A. Sard, The measure of the critical values of differentiable maps, Bull. Amer. Math. Soc. 48 (1942) 883-890.

[7] H. Schubert, Die eindeutige Zerlegbarkeit eines Knotens in Primknoten, S.-B. Heidelberger Akad. Wiss. Math.-Natur. K1. No. 3 (1949) 57-104.

[8] J. L. Synge, The first and second variations of the length in Riemannian space, Proc. London Math. Soc. 25 (1926) 247-264.

[9] J. Szenthe, On the total curvature of closed curves in Riemannian manifolds, Publ. Math. Debrecen 15 (1968) 99-105.

[10] J. A. Wolf, Spaces of constant curvature, McGraw-Hill, New York, 1967.

UNIVERSITY OF SOUTHAMPTON LEHIGH UNIVERSITY 
